\title{
Modelle einer selbstverwalteten Dritten Gewalt in Europa*
}

Das Thema „richterliche Unabhängigkeit“ ist auf nationaler wie internationaler Ebene während der zweiten Hälfte des 20. Jahrhunderts auf wachsendes Interesse gestoßen. Begonnen mit der „Allgemeinen Erklärung der Menschenrechte“ der Vereinten Nationen von 1948, die jeder Person einen Anspruch auf Rechtsschutz vor einem unabhängigen und unparteiischen Gericht gewährt, hat das Prinzip in den letzten Jahrzehnten durch Resolutionen, Empfehlungen, Erklärungen und Chartas zahlreiche Konkretisierungen erfahren. ${ }^{1}$ Zunehmend wurde über die klassischen Dimensionen richterlicher Unabhängigkeit (sachlicher, persönlicher und innerer Art) hinaus auch die strukturelle Unabhängigkeit der Justiz als Ganzes in den Blick genommen. Dies führte zur Einrichtung diverser Selbstverwaltungsorgane, welche die berufliche Organisation, also die strukturellen Rahmenbedingungen richterlicher Tätigkeit, so gestalten sollen, dass richterliche Unabhängigkeit und Unparteilichkeit gewährleistet werden können. Da eine Selbstverwaltung der Gerichte nun auch in Deutschland verstärkt diskutiert wird, kann es sich bei der Frage nach dem Ob und Wie der Umsetzung lohnen, einen Blick auf die europäischen Nachbarländer zu werfen und aus den dort in Erfahrung gebrachten Vorund Nachteilen Impulse für den Diskurs zu ziehen.

Im europäischen Raum lassen sich heute fast überall Oberste Richterräte finden, wobei jedes Land sein eigenes Modell entwickelt hat. Prinzipiell kann man bei den justiziellen Selbstverwaltungssystemen jedoch zwischen ,,Süd“- und „Nordeuropamodellen " unterscheiden: Während bei ersteren vor allem die Selbstverwaltung der richterlichen Laufbahn (Einstellung, Beförderung, Aus- und Fortbildung, Disziplinargewalt) im Vordergrund steht, liegen bei letzteren hauptsächlich die Befugnisse der Geschäfts- und Haushaltsführung (des finanziellen und administrativen Managements) in den Händen eines Obersten Richterrats. Dieser Systematisierung folgend werden zunächst die Modelle der Gerichtsverwaltungen in Italien, Frankreich und Spanien dargestellt, bevor abschließend auf die Entwicklungen in Dänemark und Großbritannien eingegangen wird.

Wenn es dem Verständnis dient, sind den Beschreibungen der Modelle konstitutionelle Besonderheiten oder Erläuterungen des historischen Kontexts vorangestellt. Die Organe der Selbstverwaltung werden anschließend vor allem hinsichtlich ihrer Funktionen und Aufgaben mit besonderem Augenmerk auf die Aspekte der Richterauswahl, der Beurteilung und Beförderung (Hierarchiestrukturen), der Disziplinarbefugnisse und des Budget(antrags-)rechts in den Blick genommen.

Ein möglicher Vergleichsmaßstab für die unterschiedlichen Systeme könnte auf supranationaler Ebene gefunden werden. Daher werden vor den einzelnen nationalen Ausgestaltungen institutioneller Unabhängigkeit Leitlinien dargestellt, die der Beirat

* Der Beitrag basiert auf einem Vortrag, der am 01. Juli 2010 an der Justizakademie NordrheinWestfalen im Rahmen einer Tagung der Vorsitzenden Richterinnen und Richter der Landesarbeitsgerichte gehalten wurde.

1 Vgl. hierzu den Überblick bei Oberto, Richterliche Unabhängigkeit - rechtsvergleichende Betrachtung ihrer institutionellen Ausgestaltung, ZRP 2004, S. $207 \mathrm{ff}$. 
der europäischen Richterinnen und Richter beim Europarat (kurz CCJE) in Empfehlungen zur Struktur und Rolle eines Obersten Richterrats bzw. eines anderen gleichwertigen unabhängigen Organs justizieller Selbstverwaltung herausgearbeitet hat und nach denen abschließend auch die vorgestellten Ländermodelle verglichen werden können.

\section{A. Europäische Leitlinien: Die Empfehlungen des CCJE aus den Jahren 2001 und 2007}

Der CCJE konkretisiert mit seinen Stellungnahmen die Empfehlung Nr. R (94) 12 aus dem Jahr 1994, die das Ministerkomitee des Europarats ,zur Unabhängigkeit, Leistungsfähigkeit und Rolle der Richter" verabschiedet hat. ${ }^{2}$ Hieraus ließen sich unter anderem folgende Minimalprinzipien ableiten:

- Richter sind unabhängig und nur dem Gesetz unterworfen;

- Richter sind unabsetzbar;

- die Auswahl und Bestimmung der Berufslaufbahn von Richtern soll ein von Regierung und Verwaltung unabhängiger Entscheidungsträger vornehmen;

- ein Oberster Richterrat muss die Unabhängigkeit der Richter garantieren.

Insbesondere seine Stellungnahmen Nr. 1 aus dem Jahr 2001 und Nr. 10 aus dem Jahr $2007^{3}$ führen die Anforderungen, die an einen Justizverwaltungsrat als Obersten Richterrat $^{4}$ gestellt werden müssen, aus. Mit ihnen sollen Leitlinien und Standards für diejenigen Mitgliedstaaten der Europäischen Union festgelegt werden, die eine Selbstverwaltung der Justiz einführen bzw. deren Regelung reformieren möchten - ohne dass mit diesen Empfehlungen die Vielfalt der bestehenden Systeme einzelner Mitgliedstaaten in Frage gestellt werden soll. Durch die Stellungnahmen soll aber zugleich auf die Bedeutung hingewiesen werden, die ein unabhängiges Organ der Justizverwaltung als Garant richterlicher Unabhängigkeit und somit auch für die Achtung des Grundsatzes der Gewaltenteilung als unabdingbarer Bestandteil jedes Rechtsstaats hat. Die in den Stellungnahmen dargelegten Anforderungen an seine Zusammensetzung und seine Funktionen sollen gewährleisten, dass ein Gleichgewicht zwischen der gesetzgebenden, der vollziehenden und der rechtsprechenden Gewalt hergestellt werden kann.

\section{Allgemeines}

Ziel eines Justizverwaltungsrats muss es sein, sowohl die Unabhängigkeit des Rechtssystems als Ganzes als auch diejenige des einzelnen Richters sicherzustellen. Der Justizverwaltungsrat soll daher neben seiner Geschäftsführungs- und Verwaltungsrolle auch als justizielles Selbstverwaltungsorgan fungieren, das den einzelnen Richtern ermöglicht, ihre Aufgaben außerhalb der Kontrolle durch Exekutive oder Legislative und

2 Abrufbar unter http://www.coe.int/t/e/legal_affairs/legal_co-operation/administrative_law_and justice/texts_\&_documents/conv_rec_res/recommendation(94)12.asp.

3 Stellungnahme Nr. $\overline{1}(2001)$ und Nr. $1 \overline{0}$ (2007) CCJE, abrufbar unter www.coe.int.

4 Im Sinne der Vereinheitlichung wird anstatt der Begrifflichkeit des Obersten Richterrats die Bezeichnung „Justizverwaltungsrat“ (im Original: Council for the Judiciary) verwendet. 
ohne unangemessenen Druck aus der Justiz selbst wahrzunehmen. Das Bestehen einer unabhängigen und unparteiischen rechtsprechenden Gewalt stellt sich als strukturelles Erfordernis eines Rechtsstaats dar, sie garantiert die Gleichheit der Bürger vor Gericht und dient dem Freiheitsschutz. Die Unabhängigkeit wird den Richtern somit nicht als Privileg, sondern im Interesse der Rechtsstaatlichkeit und der Rechtssuchenden verliehen. Daher hat der Justizverwaltungsrat auch eine effektive und qualitative Rechtsprechung gemäß Artikel 6 der Europäischen Menschenrechtskonvention zu gewährleisten, um das Vertrauen der Rechtssuchenden in die Justiz zu stärken. ${ }^{5}$

\section{Die Zusammensetzung des Justizverwaltungsrats}

Nach Ansicht des CCJE sollten weder das Parlament noch die Exekutive an dem Auswahlverfahren der Mitglieder des Justizverwaltungsrats beteiligt werden. Auch ranghohe Angehörige der Gerichtshierarchie sollten keine Einflussmöglichkeit auf den Auswahlprozess erhalten.

Um eine einseitige berufsständische Interessenvertretung zu vermeiden und die verschiedenen Meinungsströmungen der Gesellschaft angemessen $\mathrm{zu}$ berücksichtigen, wird eine gemischte Zusammensetzung des Rats empfohlen, bei der jedoch die Richterschaft die substanzielle Mehrheit bildet. ${ }^{6}$ Durch die Einbeziehung von nicht-richterlichen Mitgliedern soll die demokratische Legitimität des Rats erhöht werden. Letztere sollten vorzugsweise von nicht-politischen Organen ernannt werden; bei Einbindung des Parlaments, wird die Wahl mit qualifizierter Mehrheit favorisiert, um die Beteiligung der Opposition sicherzustellen. Die nicht-richterlichen Mitglieder können aus den Reihen herausragender Juristen, Hochschullehrer oder verdienter Mitglieder der Gesellschaft stammen. Erfahrungen auf nicht-juristischen Gebieten (Finanzwesen, Management oder Sozialwissenschaften) werden dabei als vorteilhaft erachtet. Sie sollten weder Mitglieder des Parlaments noch Angehörige der Exekutive oder Vertreter der Verwaltung sein. Hieraus folgt, dass weder der Regierungschef noch ein Mitglied der Regierung in der Eigenschaft als Minister dem Justizverwaltungsrat angehören soll. Trotz gemischter Zusammensetzung können bestimmte Aufgaben einem ausschließlich aus Richtern bestehenden Gremium vorbehalten sein, um Manipulationen oder ungebührliche Druckausübung zu vermeiden und eine parteipolitische Beeinflussung auszuschließen.

Auch eine alleinige Zusammensetzung aus Richtern wird als zulässig angesehen. ${ }^{7}$ Deren Wahl soll durch Amtskollegen und in einem Verfahren erfolgen, das eine größtmögliche Vertretung der Richterschaft garantiert. Für eine begrenzte Anzahl wird auch eine Bestellung von Amts wegen (z.B. bei den Präsidenten der Obersten Gerichtshöfe oder anderer Rechtsmittelinstanzen) als zulässig anerkannt. Zudem wird begrüßt, die Richtervereinigungen in angemessenem Umfang an den Wahlen zu beteiligen. Um eine Politisierung der Wahlen zu vermeiden, soll das „Werben um Stimmen“ jedoch in einem vom Rat erlassenen Verfahren erfolgen.

5 Vgl. Stellungnahme Nr. 10 (Fn. 3), II., 8.-14.

6 Vgl. Stellungnahme Nr. 10 (Fn. 3), III., A.-C.

7 Vgl. Stellungnahme Nr. 10 (Fn. 3), III., A., 16. und 17. 
Die Ernennung der (richterlichen oder nicht-richterlichen) Mitglieder soll anhand ihrer Kompetenz, ihrer Erfahrung, ihres Verständnisses vom Gerichtsalltag und ihres Umgangs mit der Unabhängigkeit erfolgen.

\section{Die Befugnisse des Justizverwaltungsrats}

Primäre Aufgabe jedes Justizverwaltungsrats ist es, die richterliche Unabhängigkeit und die Effektivität der Justiz zu schützen und zu fördern. Daher sollten vor allem die Auswahl, Ernennung und Beförderung der Richter in seine Zuständigkeit fallen und unabhängig von Legislative oder Exekutive sowie im Hinblick auf die Auswahlkriterien völlig transparent erfolgen. Des Weiteren wird empfohlen, die Zuständigkeit für Disziplinarmaßnahmen, das Haushaltsrecht, die Verwaltung und Geschäftsführung der einzelnen Gerichte sowie die Vertretung der Justiz nach außen auf den Justizverwaltungsrat zu übertragen. ${ }^{8}$

Hinsichtlich der Richterauswahl, -ernennung und -beförderung sei es zwar zulässig, dass die Ernennung oder Beförderung als offizielle Amtshandlung durch den Staatsoder Regierungschef vorgenommen werde. Dies müsse jedoch ein formaler Akt bleiben, bei dem das Staatsoberhaupt an die Vorschläge des Justizverwaltungsrats gebunden bleibt. Nicht ausreichend sei es, den Rat lediglich um eine Stellungnahme zu einem Ernennungsvorschlag zu bitten, weil bereits die Tatsache, dass der Vorschlag von einer politischen Instanz stamme, das Ansehen der richterlichen Unabhängigkeit negativ beeinträchtige.

Auch das Disziplinarrecht - das als Garant des bürgerlichen Freiheitsschutzes fungiert - müsse in den Händen des Justizverwaltungsrats (als einem Organ frei von politischer Einflussnahme) liegen; die Mitwirkung des Staats- bzw. Regierungschefs, des Justizministers oder eines Vertreters der Exekutive am Disziplinarverfahren wird ausgeschlossen.

Der Justizverwaltungsrat sollte umfassende Haushaltsbefugnisse hinsichtlich der Aufstellung und Verwaltung des Justizhaushaltes sowie Befugnisse in Bezug auf die Verwaltung und Geschäftsführung der Gerichte (wenn auch nicht zwingend die Alleinverantwortung) zwecks einer Verbesserung der Qualität der Justiz haben.

\section{B. Das italienische Modell}

Das italienische Modell justizieller Selbstverwaltung wird häufig als das Modell bezeichnet, das den höchsten Grad an richterlicher Unabhängigkeit garantiert. Es zeichnet sich vor allem durch einen starken und autonomen Richterrat aus, der als fundamentale Voraussetzung für die Gewährung und Stärkung einer unabhängigen Justiz gesehen wird.

Die Wurzeln des Strebens nach einer unparteilichen, möglichst unbeeinflussten Justiz finden sich in der jüngeren italienischen Geschichte: ${ }^{9}$ Vor allem die Schwäche staatli-

8 Vgl. Stellungnahme Nr. 10 (Fn. 3), V., A.-C., E. und F.

9 Vgl. hierzu Mariuzzo, Die Selbstverwaltung der ordentlichen Richter in Italien - der Oberste Rat der Richterschaft, DRiZ 2001, S. 162 f. 
cher Institutionen, insbesondere der Justiz, beschleunigte die Ausbreitung des totalitären Regimes Mussolinis. Nach Beendigung der faschistischen Diktatur war die neue Republik daher bemüht, die Verwaltungsmacht des Justizministers zu beseitigen, die zuvor dafür genutzt worden war, Richter und Staatsanwälte durch Auswahl, Ernennung und Beförderung politisch zu kontrollieren. Die seit 1947 bestehende italienische Verfassung sieht daher für die Verwaltung der ordentlichen Gerichtsbarkeit einen Obersten Richterrat (Consiglio Superiore della Magistratura; kurz CSM) und eine unabhängige Staatsanwaltschaft vor. In ihr finden sich zudem zahlreiche Garantien für eine justizelle Unabhängigkeit: So erklärt Art. 104 die Judikative zu einem von den anderen Staatsgewalten unabhängigen und autonomen Stand; Art. 105 überträgt die Personalverwaltung der Justiz (d.h. das Recht zur Einstellung, Versetzung, Beförderung und Ämterzuweisung sowie das Disziplinarrecht) dem Obersten Richterrat und betont hierdurch die autonome Stellung der Judikative gegenüber der Exekutive. Deren Einflussmöglichkeit auf den beruflichen Aufstieg und die allgemeine Stellung der Richter wurde dadurch beseitigt. Art. 101 bindet den Richter schließlich an das Gesetz und emanzipiert somit die Dritte Gewalt außerhalb des ordnungsgemäßen Gesetzgebungsverfahrens auch von Einflussnahmen der Legislative.

\section{Zusammensetzung des CSM}

Die Zusammensetzung des CSM ist in den Grundzügen konstitutionell verankert. Er besteht zurzeit aus 27 Mitgliedern, von denen gemäß Art. 104 der Verfassung drei Mitglieder kraft Amtes sind: der Präsident der Republik als Vorsitzender sowie der Erste Präsident und der Generalstaatsanwalt des Kassationsgerichts. Die übrigen 24 Mitglieder werden zu zwei Dritteln von den Richtern und Staatsanwälten selbst und zu einem Drittel durch das Parlament in gemeinsamer Sitzung und mit qualifizierter Mehrheit (3/5 der Anwesenden) von Abgeordnetenhaus und Senat gewählt. Die sogenannten Laienmitglieder (im Gegensatz zu den Mitgliedern ,in Robe“) rekrutieren sich aus den Reihen der ordentlichen Universitätsprofessoren und verdienter Rechtsanwälte mit mehr als 15 Jahren Berufserfahrung. Um den Eindruck einer unabhängigen Justiz nicht zu gefährden, dürfen die Laienmitglieder während ihrer Amtsdauer weder als Anwalt eingetragen sein noch dem Parlament oder der Regierung bzw. der Verwaltung angehören. Von den 16 richterlichen Mitgliedern sind zwei Angehörige des Kassationsgerichts bzw. der Generalstaatsanwaltschaft beim Kassationsgericht, vier Mitglieder rekrutieren sich aus den verschiedenen Staatsanwaltschaften bei den Landgerichten (Procure della Repubblicca preso $i$ Tribunali) oder den Generalstaatsanwaltschaften bei den Oberlandesgerichten (Procure della Repubblica presso le Corti d'appello), die übrigen zehn sind Richter an Landgerichten (tribunali) oder Oberlandesgerichten (Corti d'appello). Die Amtszeit der Ratsmitglieder beträgt vier Jahre. Da eine unmittelbare Wiederwahl nicht zulässig ist, wird der CSM alle vier Jahre vollständig neu zusammengesetzt.

Bis auf die drei Mitglieder kraft Amtes trifft die Verfassung keine genauen Angaben zur Größe des Richterrats, lediglich das Verhältnis der Mitglieder ( $2 / 3 \mathrm{zu} 1 / 3)$ ist festgeschrieben. Es obliegt daher dem einfachen Gesetzgeber, die Anzahl der wählbaren Mitglieder und den Wahlmodus festzulegen. Beides war erst 2002 Gegenstand einer Reform, in der sowohl die Anzahl der Mitglieder von 33 (10 plus 20 plus 3) auf 27 reduziert 
wurde und das Wahlsystem von einer modifizierten Verhältniswahl in ein Mehrheitswahlsystem geändert wurde. Bis 2002 wurden die richterlichen Mitglieder noch von den vier italienischen Richter- und Staatsanwaltschaftsorganisationen ,gestellt": So wurden - bis auf die Wahl der beiden Kassationsgerichtsangehörigen - vier Wahlkreise gebildet, für die jeweils von den Berufsverbänden Listen erstellt wurden. Pro zu wählendem Mitglied durfte je eine Stimme abgegeben werden. Die Kandidaten mit den proportional meisten Stimmen wurden in den CSM gewählt. Die vier Wahlkreise wurden dadurch gebildet, dass ihnen die 26 Berufungsgerichtsbezirke jeweils durch Los zugeordnet wurden, so dass sich die Zusammensetzung der Wahlkreise bei jeder Wahl änderte. An diesem System wurde kritisiert, dass sich keine Wahlkreise herausbilden konnten, die sich auf homogene geografische Gebiete bezogen. Das Gesetz Nr. 44 vom 28. März 2002 löste das Konzept der „regionalen“ Wahlkreise auf und ersetzte es durch drei gesetzlich festgelegte, nach sachlichen Kriterien eingeteilte Wahlkreise: je einer für die Richter am Kassationsgericht, die Staatsanwaltschaft und die Richter an den Land- und Oberlandesgerichten. Jeder Richter bzw. Staatsanwalt darf drei Stimmen (eine pro Wahlkreis) abgeben. Gewählt sind die Kandidaten, die die meisten Stimmen auf sich vereinigen können. Auch das neue Wahlsystem ist nicht frei von Kritik: So wird ihm vorgehalten, seine eigentliche Intention - nämlich die Reduzierung des Einflusses der Richterorganisationen durch das Abschaffen des Rechts zur Aufstellung der Kandidatenlisten - verfehlt und sogar in das Gegenteil verkehrt zu haben: Die Folge sei eine Politisierung der Richterschaft, die stärker denn je der „Logik der politischen Mehrheit" folge, da nur noch Kandidaten, die die Unterstützung von einflussreichen Gruppen finden, gewählt werden können. ${ }^{10}$

Der Vorsitz des Rats liegt beim Präsidenten der Republik, während der Vizepräsident vom CSM selbst aus den Reihen der parlamentarisch legitimierten Mitglieder gewählt wird. Der Vizepräsident, der zeitgleich dem Präsidialausschuss vorsitzt, hat die Aufgaben, die Tätigkeiten des Rats sowie die Ausführung seiner Beschlüsse zu fördern und die Haushaltsmittel zu verwalten. Ferner vertritt er den Präsidenten bei dessen Abwesenheit und kann an ihn delegierte Funktionen wahrnehmen. Der Justizminister hingegen ist nicht Mitglied des Obersten Richterrats. Er hat jedoch das Recht, an seinen Sitzungen teilzunehmen und Erklärungen abzugeben. Das Zusammenspiel dieser drei Akteure spiegelt die institutionelle Verflechtung des CSM mit anderen Staatsorganen wider: Dieser trifft zwar in Eigenverantwortung die meisten Entscheidungen, welche die Justiz betreffen, umsetzen kann die Beschlüsse des Rats jedoch nur der Präsident der Republik (in seiner Eigenschaft als Staatsoberhaupt und nicht als Vorsitzender des Richterrats). Alle Umsetzungsakte des Präsidenten müssen zuletzt vom Justizminister - als Teil der Regierung - gegengezeichnet werden.

\section{Funktion und Aufgaben des CSM}

Die Bündelung der Zuständigkeiten in den Händen des CSM, die den Status von Richtern berühren, soll die Unabhängigkeit und die Autonomie der Justiz garantieren. Eine

10 Vgl. Salvi, Selbstverwaltung und Verfassungsrecht: Die italienische Erfahrung, KritV 2008, S. $368 \mathrm{f}$. 
als absolut verstandene Selbstverwaltung ist hiermit jedoch nicht gemeint: Insbesondere das Verfassungsgericht hat immer wieder in seinen Entscheidungen betont, dass der CSM als nur eines von mehreren Verfassungsorganen zur Kooperation verpflichtet ist und sich in das Staatsgefüge eingliedern muss. Dies betrifft speziell die Zusammenarbeit mit dem Justizminister, dem die Verfassung in Art. 110 die Aufgabe des Aufbaus und der Verwaltung des Justizwesens zuweist und der das ordnungsgemäße Funktionieren der Justiz dem Parlament gegenüber verantworten muss. ${ }^{11}$ Solche komplexen institutionellen Verflechtungen lassen es angebracht erscheinen, statt von Selbstverwaltung von einer autonomen Verwaltung zu sprechen. Seine Funktion als Verwalter der Rechtsprechung umfasst in erster Linie die Personalverwaltung der Richterschaft und konkretisiert sich in Einstellung, Zuweisung, Versetzung, Beförderung und Disziplinargewalt.

Die Richterernennung ${ }^{12}$ erfolgt gemäß Art. 106 der Verfassung über ein landesweites, öffentliches Zugangs- und Auswahlverfahren, Concorso genannt. Man unterscheidet zwischen einem Concorso für Rechtsreferendare und einem Concorso für Richter an Landgerichten. Ersterer deckt ca. $90 \%$ der frei werdenden Personalstellen und setzt neben einem abgeschlossenen Jurastudium - eine Vorprüfung in den Kernfächern voraus. Das 2001 geschaffene Concorso-Verfahren für Richter an Landgerichten wurde für Anwälte unter 45 Jahren geschaffen, die sich erst spät und durch verdienstvolle Leistungen für den Richterberuf entschieden haben. Beide Concorsi schließen mit einem Examen ab. Die Concorsi werden auf Beschluss des CSM vom Justizministerium ausgeschrieben, wobei beide Institutionen bei der Festlegung der Stellenanzahl zusammenarbeiten. Der CSM ernennt dann eine Prüfungskommission, die eine auf den Leistungen der Kandidaten basierende Rangliste erstellt, welche vom CSM bestätigt wird. Kandidaten, die den Concorso erfolgreich absolviert haben, werden zu Rechtsreferendaren ernannt und einer erstinstanzlichen Justizbehörde für ein meist zweijähriges Praktikum zugewiesen, das vom CSM geleitet, organisiert und kontrolliert wird. Nach der Ausbildungszeit weist der Oberste Justizrat nach der Reihenfolge der Concorso-Rangliste den Kandidaten freie Plätze in den erstinstanzlichen Gerichten zu. Der CSM ist somit maßgeblich an allen Stadien der Ausbildung und Auswahl von Rechtsreferendaren beteiligt und alleinzuständig für die Ernennung der einzelnen Richter und Staatsanwälte.

Auch die Beförderung und die Ämterzuweisung erfolgt ausschließlich durch den CSM, wobei sich das italienische System der offenen Laufbahn maßgeblich von Laufbahnstrukturen anderer Länder unterscheidet. ${ }^{13}$ Art. 107 Abs. 3 der Verfassung legt fest, dass sich die einzelnen Richter nur aufgrund ihrer Ämter unterscheiden. Dies hat maßgeblichen Einfluss auf das italienische Beförderungssystem, das den Dienst- und somit Beförderungsgrad von der ausgeübten Funktion abkoppelt und eine automatische Beförderung nach Dienstalter - vorbehaltlich schuldhafter Vergehen - vorsieht. Funktion und Beförderungsrang entwickeln sich somit nicht notwendigerweise parallel, sondern sind immer getrennt voneinander zu betrachten.

11 Dem Justizminister sind jedoch in der Hauptsache nur noch die Aufgaben der materiellen Ausstattung der Gerichte sowie der Anstellung des nicht-richterlichen Personals übertragen.

12 Vgl. Salvi, Das italienische System „offener Rollen“: Beurteilungen im Rahmen autonomer Laufbahnstrukturen einer selbstverwalteten Justiz, KritV 2008, S. $423 \mathrm{ff}$.

13 Vgl. hierzu Oberto (Fn. 1), S. 209. 
Das gesetzlich vorgeschriebene Dienstalter zur Ernennung zum Richter am Landgericht beträgt zwei Jahre ab Dienstantritt als Rechtsreferendar. Nach elf Jahren können Richter am Landgericht in den Rang von Richtern am Berufungsgericht und nach weiteren sieben Jahren von Richtern am Kassationsgericht ernannt werden. Acht Jahre danach kann die Ernennung zu höheren Leitungsämtern erfolgen. Ist das erforderliche Dienstalter erreicht, wird die Beförderung nach Anhörung des zuständigen Gerichtsrats, der regelmäßig (alle vier Jahre) im Laufe der Dienstzeit Beurteilungen der richterlichen Arbeit vornimmt, vom CSM beschlossen. Da die Beurteilungen zu $90 \%$ positiv ausfallen, wird in der Regel die nächste Laufbahnstufe mit der entsprechend höheren Besoldung automatisch erreicht. Die durchweg positiven und durch Standardklauseln wenig aussagekräftigen Beurteilungen gaben - auch wegen des im Ausland als z.T. desolat empfundenen Zustands der italienischen Justiz ${ }^{14}$ - immer wieder Anlass zur Kritik. Daher wurde 2007 eine Reform des Beurteilungswesens angegangen, die auch den Inhalt und die Art richterlicher Entscheidungen mit in die Bewertungsgrundlage mitaufnahm. Die Beurteilungen sollten dadurch detaillierter, transparenter und überzeugender werden.

Neben der automatischen Beförderung bedeutet die Trennung von Dienstgrad und ausgeübtem Amt auch, dass die Beförderung nicht mit der tatsächlichen Zuteilung zu einer dem erreichten Dienstgrad entsprechenden Stelle korreliert, sondern als unmittelbare Folge lediglich die entsprechende Besoldung hat. So kann ein Richter, der für die Ernennung zum Berufungsrichter als geeignet befunden wurde, weiterhin ohne zeitliche Befristung in seinem Amt bleiben, auch wenn dieses einem niedrigeren Dienstgrad entspricht. Der Vorteil dieser Einheitlichkeit der Richterämter wird in der Unterbindung jeglicher Hierarchiestrukturen mit den möglicherweise einhergehenden Abhängigkeitsformen und der bestmöglichen Förderung des Einsatzbereiches gesehen. Die Richter sollen sine spe ac metu, ohne Hoffnung und Furcht in Bezug auf ihre Karriere nur anhand des Gesetzes entscheiden. ${ }^{15}$

Der CSM hat ebenfalls die Disziplinargewalt über die Richter und Staatsanwälte. Er kann jedoch nicht von sich aus Disziplinarmaßnahmen einleiten, das Initiativrecht hierfür liegt beim Generalstaatsanwalt und dem Justizminister. Eine eigene Disziplinarabteilung trifft Entscheidungen per Mehrheitsbeschluss, Stimmengleichheit führt zu einer für den Betroffenen günstigeren Entscheidung. Der Großteil der in Italien eingeleiteten Disziplinarverfahren betrifft den Vorwurf der überlangen Verfahrensdauer bzw. der Verfahrensverzögerung sowie ein ungenügendes Engagement bei der Aufgabenerfüllung. Entgegen dem Vorwurf der „Hausjustiz“ werden jährlich ca. $10 \%$ aller Richter und Staatsanwälte (das entspricht einer tatsächlichen Zahl von 900) einem Disziplinarverfahren unterworfen, ca. $40 \%$ hiervon auch sanktioniert. ${ }^{16}$

Zum weiteren Aufgabenfeld des CSM gehört die Organisation der Justizbehörden. Gemeinsam mit dem Justizminister obliegt es ihm, die Gerichtspräsidenten der einzelnen Gerichte und die Leiter der Staatsanwaltschaften zu benennen. Um die Autonomie

14 Im Durchschnitt beträgt die Zeit bis zu einer erstinstanzlichen Entscheidung drei Jahre und bis zu 10 Jahre bis zum Abschluss des Verfahrens (Stand: 2002). Rund 12000 Menschenrechtsbeschwerden sind beim Europäischen Gerichtshof für Menschenrechte unter Berufung auf eine überlange Verfahrensdauer (Verstoß gegen Art. 6 Abs. 1 EMRK) anhängig.

15 Vgl. Pivetti, Gewaltenteilung in Italien, Betrifft Justiz 1999, S. 134 ff.

16 Vgl. Salvi (Fn. 12), S. 432 f. 
der Personalentscheidungen nicht zu untergraben, ist dem CSM auch finanzielle Unabhängigkeit zuerkannt worden. Der Präsidialausschuss des Rats verwaltet daher das Budget, das im Staatshaushalt für die Justiz bereit gestellt wird. Ein echtes Budgetantragsrecht besitzt der CSM jedoch nicht.

Beschränkt wird die Macht des CSM durch die Befugnisse der sog. Gerichtsräte (Consigli giudiziari), die - vergleichbar mit den deutschen Gerichtspräsidien - „basisnäher" ebenfalls mit Justizverwaltungsaufgaben betraut sind. ${ }^{17}$ Sie sind das Bindeglied zwischen den Gerichten und dem CSM. Gerichtsräte sind für die einzelnen Berufungsgerichte - seit 2008 auch für Kassationsgerichte - vorgesehen und bestehen aus dem Präsidenten und dem Generalstaatsanwalt des Gerichts sowie fünf weiteren Magistrati, die alle zwei Jahre von den dem Gericht zugeordneten Richtern gewählt werden. Sie sind vor allem für die Beurteilungen der Richter und die Geschäftsverteilung (mit-)zuständig.

\section{Reformvorhaben}

Das Verhältnis von Justiz und Politik ist und bleibt in Italien ein Punkt ständiger Auseinandersetzung. Verschärft haben sich diese Verhältnisse unter der Regierung Berlusconi, ${ }^{18}$ der offen gegen die „roten Roben“ vorgeht. So verwundert es auch nicht, dass die angestrebten Verfassungsreformen ${ }^{19}$ vor allem die Zusammensetzung und die Befugnisse des CSM im Blick haben. Angestrebt ist sowohl, den Anteil der vom Parlament gewählten Mitglieder auf $50 \%$ zu erhöhen, als auch eine Trennung von Richter- und Staatsanwaltschaft mit dem Ziel herbeizuführen, die Staatsanwaltschaft der Exekutive $\mathrm{zu}$ unterstellen. Insbesondere das deutsche Modell dient hierbei als Vorbild für Reformbemühungen. Insgesamt soll ein größerer Einfluss auf die Personalentscheidungen innerhalb der Justiz ausgeübt werden können, da das italienische Justizsystem ,,veraltet und ineffizient" sei. Bisher haben sich jedoch noch keine dieser Reformen verwirklichen lassen.

\section{Fazit}

Zusammenfassend lässt sich sagen, dass der CSM im institutionellen Zusammenspiel der Staatsgewalten einen wesentlichen Schutz für die Autonomie der Justiz als Ganzes und der Unabhängigkeit des einzelnen Richters bietet, auch weil er als Vertretung der Dritten Gewalt nach außen anerkannt ist. Die Beteiligung der einzelnen Gerichtsebenen ermöglicht ihm - in Grenzen - eine autonome Verwaltung ,,von unten “. Die Zusammensetzung aus Richtern und parlamentarisch-legitimierten Laien sowie die Notwendigkeit der Zusammenarbeit mit dem Justizministerium bei der Umsetzung der eigenen Beschlüsse zeigen, dass der CSM in das staatliche Gewaltgefüge eingebettet und legitimiert ist und so die Balance der Staatsgewalten aufrecht erhalten wird. Kritisiert wird

17 Vgl. Salvi (Fn. 10), S. 377 ff.

18 Vgl. Castillo, Die neue Bedrohung für Italiens Justiz, Betrifft Justiz 2005, S. 118 ff.; Senf, Italien - die neue Dreistigkeit, Betrifft Justiz 2002, S. 375 ff.

19 Vgl. hierzu Wittreck, Die Verwaltung der Dritten Gewalt, 2006, S. 561 f. 
an diesem Modell jedoch die auf das System der offenen Laufbahn und fehlender Leistungsanreize zurückgeführte Nivellierung nach unten, die einem Rezipieren des Systems zumindest vorsichtige Vorbehalte entgegensetzt. Auch die je nach Regierung stärker hervortretende Politisierung der Richterschaft und des CSM wird argwöhnisch beäugt - insbesondere vor dem Hintergrund, dass durch die Machtkonzentration auf den CSM auch neue Gefährdungspotentiale für den einzelnen Richter geschaffen wurden.

\section{Das französische Modell}

In Frankreich wird deutlich, dass es das Staatsverständnis ist, das die Form der justiziellen Selbstverwaltung entscheidend mitbestimmt. ${ }^{20}$ Bereits in der ersten französischen Verfassung von 1791 war zwar die formale Gewaltenteilung, d.h. die Trennung von Judikative, Legislative und Exekutive als Voraussetzung für bürgerliche Freiheit anerkannt, jedoch nicht die Garantie der Unabhängigkeit der Judikative von den anderen Gewalten. Dies war zurückzuführen auf ein durch Korruption begründetes generelles Misstrauen der Bevölkerung gegenüber der Richterschaft in der postrevolutionären Zeit, was sich am eindrucksvollsten in der Formulierung von Montesquieu wiederfindet, dass der Richter lediglich bouche de la loi, der Mund des Gesetzes sei. Richter wurden verstanden als Verkünder, nicht als Interpreten der Gesetze. Ihre Autonomie war dadurch nicht erforderlich, vielmehr betrachtete man die Justiz als der Exekutive zugeordnet. Eine historisch gewachsene Vorstellung, die sich erst in jüngerer Zeit durch ein modernes Verständnis von Gesetzen und deren Auslegungs- und Anwendungsoffenheit gewandelt hat. Das Misstrauen und das Verständnis von Gewaltenteilung als Schutz der Exekutive und Legislative vor der Dritten Gewalt begründete auch das Interesse vieler französischer Staatsoberhäupter, die Kompetenzen der Richter genau zu überwachen und festzulegen, um die Justiz keinen zu großen Einfluss erlangen zu lassen. Dies setzte sich von Napoleon bis hin zu de Gaulle in die V. Republik durch.

Mit dem neuen Verständnis von Rechtsanwendung kam jedoch die Erkenntnis hinzu, dass Richter vor äußeren Einflüssen geschützt werden müssen, welche die Orientierung der Rechtsprechung allein am Gesetz gefährden. Nun wurde zum ersten Mal die (organisatorische) Zuordnung zur Exekutive als Bedrohung der Unparteilichkeit gesehen. Ein erster Schritt hin zur als erforderlich betrachteten richterlichen Unabhängigkeit war im Jahr 1948 die verfassungsrechtliche Garantie des Conseil Supérieur de la Magistrature (Hoher Richterrat, fortan als CSM bezeichnet) als neue Zentralinstanz der französischen Rechtspflege. ${ }^{21}$ Doch erst 1958 wurde die richterliche Unabhängigkeit durch Art. 64 auch in der Verfassung garantiert. Das Misstrauen gegenüber der Judikative ist darin noch in zweierlei Hinsicht erkennbar: Zum einen wird die Judikative nicht als eigenständige Gewalt (pouvoir) bezeichnet, sondern nur die Unabhängigkeit ihrer Autorität (autorité judiciaire) gewährleistet. Zum Anderen erfolgt trotz einer in Grundzügen anerkannten Selbstverwaltung noch die Rückbindung der Dritten Gewalt an die Exekutive, da die Verfassungsregelung nicht die Dritte Gewalt selbst zum Subjekt der

20 Vgl. Horn, Einführung in die Rechtswissenschaft und Rechtsphilosophie, 4. Auflage, 2007, Rn. 453.

21 Art. 83 der Verfassung von 1846; einfachgesetzlich erwähnt wurde der CSM jedoch bereits 1883. 
Regelung macht, sondern die Garantie ihrer Autorität dem Präsidenten auferlegt. ${ }^{22}$ Der Conseil Supérieur de la Magistrature hat lediglich die Aufgabe, ihn dabei zu unterstützen. ${ }^{23}$ Dies erklärt sich aus der erweiterten Rolle des Präsidenten seit der V. Republik: Danach soll alle staatliche Gewalt - auch die rechtsprechende - über den Präsidenten, als den durch Direktwahl vom Volk bestimmten Vertreter, vermittelt werden. ${ }^{24}$ Als oberster Vertreter des Volkes obliegt es ihm, die Staatsgewalten zu harmonisieren, er ist der arbitre (Schiedsrichter) aller staatlichen Institutionen.

\section{Zusammensetzung des Conseil Supérieur de la Magistrature}

Der Conseil Supérieur de la Magistrature soll zur Gewährleistung der Unabhängigkeit der Dritten Gewalt beitragen. Er besteht gemäß Art. 65 der Verfassung aus zwei Kammern: eine für die magistrats du siège (Richter) und eine für die magistrats du parquet (Staatsanwälte). Zuständig ist der CSM für die gesamte ordentliche Gerichtsbarkeit. Die Verwaltungsgerichtsbarkeit hingegen ist hiervon nicht erfasst.

Die für die Richter zuständige Kammer des CSM besteht aus 15 Mitgliedern. Davon werden 6 aus den Reihen der Richter und Staatsanwälte bestellt, wobei das Verhältnis $5 \mathrm{zu} 1$ beträgt. 6 Plätze sind für nicht-richterliche Mitglieder vorgesehen, die beiden Kammern gleichzeitig angehören. Je zwei werden dabei vom Präsidenten der Republik, dem Präsidenten der Nationalversammlung (Assemblé Nationale) und dem Präsidenten des Senats bestimmt. Sie rekrutieren sich aus dem Kreis verdienstvoller Personen (z.B. Universitätsprofessoren oder hohe Amtsinhaber anderer Staatsorgane) und dürfen weder dem Parlament noch der Gerichtsbarkeit oder der Verwaltung angehören. Weiter werden der Kammer ein Mitglied des Staatsrats und ein Rechtsanwalt (gewählt vom Dachverband der französischen Anwaltskammern) zugeteilt. Hinzu kommt ihr Vorsitzender, dessen Mitgliedschaft kraft Amtes erfolgt. Bis zur großen Reform des CSM durch Verfassungsänderung im Jahre $2008^{25}$ hielt der Präsident der Republik das Amt des Vorsitzenden inne. Da der Richterrat für die Ernennung der höchsten Richterämter zuständig ist, spiegelte sich in dieser Regelung der Einfluss des Präsidenten auf die Zusammensetzung der Richterschaft wider. Durch seine Person sollte die Einsetzung der Richter demokratisch legitimiert werden. Als festes Mitglied des Rats war zudem der Justizminister kraft Amtes als Vize-Präsident vorgesehen, er sollte als Vertreter der Exekutive eine Verbindung zwischen ihr und der Justiz herstellen.

Seit 2008 ist nun gemäß Art. 65_2 der Präsident des Kassationsgerichts (Cour de Cassation) Vorsitzender des Rats. Dies wird als Zugewinn für die Unabhängigkeit und als weitergehende Verwirklichung des Gewaltenteilungsgrundsatzes verstanden, da der Präsident der Republik als oberstes Organ der Exekutive und von den Parteien abhängig

22 So heißt Art. 64 im Wortlaut: „Der Präsidenten der Republik ist Garant der Unabhängigkeit der gerichtlichen Autorität“ („Le Président de la République est garant de l'indépendance de l'autorité judiciaire").

23 „Il est assisté par le Conseil Supérieur de la Magistrature” (Art. 64_2).

24 Nach De Gaulle gibt es keine Gewalt militärischer, ziviler, behördlicher oder justizieller Art, die von einer anderen Person ausgeht als dem Präsidenten.

25 Vgl. Alt, Europäische Tendenzen: Herausforderungen und notwendige Entscheidungen, KritV 2008, S. 362. 
nicht im Sinne der justiziellen Autorität handeln, sondern die Interessen der Exekutive vertreten könnte. Auch ist der Justizminister nicht mehr als Vertreter des Ratsvorsitzenden vorgesehen. Er und der Präsident der Republik dürfen nur noch als Zuhörer an Sitzungen des CSM teilnehmen, bei Disziplinarverfahren sind sie gänzlich ausgeschlossen.

\section{Kompetenzen des Conseil Supérieur de la Magistrature}

Die Kompetenzen des CSM reichen zwar nicht an die seines italienischen Gegenstücks betreffend der Justiz- und Gerichtsverwaltung heran, seine Befugnisse erstrecken sich jedoch über die Benennung der Richter der obersten Gerichtshöfe, die Mitwirkung bei der Ernennung der übrigen Richter bis zur Disziplinargewalt über die gesamte Richterschaft. ${ }^{26}$

Bezüglich der Ernennung (und somit zugleich auch der Beförderung) der Richter am Kassationsgerichtshof (als höchstes ordentliches Gericht Frankreichs), der Ernennung des Präsidenten an den Appellationsgerichtshöfen sowie der Ernennung der Präsidenten an den Tribunaux de grand instance (Großinstanzgerichten) besitzt der CSM seit 1993 gemäß Art. 65_4 der Verfassung das Initiativrecht. Insgesamt betrifft dies ca. 180 Richterstellen. Diese Vorschläge werden vom Präsidenten der Republik gegengezeichnet, er kann jedoch keinen anderen Kandidaten als den vom Rat vorgeschlagenen ernennen. In der Praxis versucht der CSM jedoch bereits im Vorfeld eine Einigung mit dem Präsidenten zu erreichen. Anwärter für die frei werdenden Stellen können sich beim CSM bewerben, der dann ihre Eignung prüft und sich intern auf einen Kandidaten festlegt. Das Initiativrecht für die übrigen Richterstellen obliegt dem Justizminister, der Oberste Richterrat hat hierzu jedoch sein Einvernehmen zu erklären (sog. avis conforme). ${ }^{27}$ Bei einer ablehnenden Haltung kann der Rat jedoch keinen eigenen Kandidaten vorschlagen, die Stelle bleibt vakant, bis eine passende Alternative vom Justizministerium vorgeschlagen wird.

Gemäß Art. 65_5 der Verfassung besitzt die Richterkammer des CSM die Disziplinargewalt über die Richterschaft. ${ }^{28}$ Die Sicherung der richterlichen Unabhängigkeit soll durch die Entscheidung über Disziplinarmaßnahmen durch Ihresgleichen erfolgen. Das Disziplinarverfahren kann nur von einer zur Anrufung der Kammer berechtigten Person erfolgen: Als solche kommen der Justizminister, seit 2001 die Präsidenten der Appellations- und Großinstanzgerichte sowie seit 2008 auch der einzelne, durch eine richterliche Entscheidung benachteiligte Bürger in Betracht. Vor allem das Einleitungsrecht der Gerichtspräsidenten wurde als begrüßenswerter Schritt in Richtung einer effektiven

26 Vgl. Wittreck (Fn. 19), S. 571 ff.

27 Zuvor war der CSM bei der Ernennung der Richter nur berechtigt, eine Stellungnahme abzugeben, die aber keine Bindungswirkung entfaltete. Weiterhin nur beratende Empfehlungen kann der CSM zur Einstellung von Staatsanwälten abgeben.

28 Vgl. Martin, Le Conseil supérieur de la magistrature et l'indépendance des juges, RDP 1997, S. 775 . 
Selbstkontrolle und weg von der Unterordnung unter die Exekutive gesehen. ${ }^{29}$ Negative Entscheidungen (Versetzungen, Tadel, Entzug von Funktionen) können vor dem Staatsrat überprüft werden, da die Einleitung eines Disziplinarverfahrens durch den Justizminister immer auch eine Beeinflussung der Exekutive auf die Judikative darstellen kann.

\section{Zusammenfassung}

Der Conseil Supérieur de la Magistrature spielt bei der Sicherung der richterlichen Unabhängigkeit in Frankreich eine große Rolle, auch wenn die ihm verliehenen Befugnisse hinter anderen Richterräten zurückbleiben und nur einen Teil der Richterschaft direkt betreffen. Dies korrespondiert mit einer deutlich stärkeren Stellung der Exekutive in den Fragen der Verwaltung der Judikative. So obliegt dem Justizminister die Aufsicht über die ordentlichen Gerichte, die in Frankreich z.T. auch in Teile der Rechtsprechungstätigkeit hineinreicht. Zudem obliegt ihm die Gerichtsverwaltung im engeren Sinne (Ausstattung etc.). Doch zeigt insbesondere die fortdauernde Beschränkung der Einwirkungsmöglichkeiten des Präsidenten, dass die Wertschätzung einer unbeeinflussten Rechtsprechung steigt ${ }^{30}$ Allerdings wird der Rat immer noch primär als Zwischeninstanz und nicht als Teil der Judikative gesehen: Er soll den Dialog und die Zusammenarbeit zwischen den beiden Gewalten Exekutive und Judikative fördern. Von einer eigenen, genuinen Selbstverwaltung kann und sollte deswegen nicht gesprochen werden. Dies wird darin sichtbar, dass die Anzahl der Richter im CSM kontinuierlich verringert wurde und diese sich seit 2008 in der Minderheit befinden. Die Zusammensetzung des CSM aus den verschiedenen Tätigkeitsfeldern soll die Zugänglichkeit zum Rat sichern und eine Kooptation vermeiden.

\section{Das spanische Modell}

Die spanische Verfassung regelt in den Artt. $117 \mathrm{ff}$. Funktion und Ausgestaltung der „rechtsprechenden Gewalt“ (Art. 117 Abs. 1), die damit eine Sonderstellung erhält. ${ }^{31}$ Die Idee der justiziellen Selbstverwaltung ist hierbei durch drei sich ergänzende Organe auf unterschiedlichen Ebenen und mit unterschiedlichen Befugnissen verwirklicht: Durch den Allgemeinen Justizrat oder „Obersten Rat der Gerichtsbarkeit“ (Consejo General del Poder Judicial - CGPJ), durch die Verwaltungskammern am Obersten Gerichtshof (Kassationsgericht), am Nationalen Gerichtshof und an den Oberen Gerichtshöfen der autonomen Regionen (Salas de Gobierno) sowie durch die Generalversammlung der Richter (Junta de Jueces). Dem Justizminister verbleiben in diesem System

29 Dass die Verpflichtung zur Selbstkontrolle auch angenommen wird, zeigt sich darin, dass 2008 erstmals mehr Disziplinarmaßnamen durch die Gerichtspräsidenten als durch den Justizminister eingeleitet wurden.

30 Dieser hatte bis 1993 neben dem Ratsvorsitz zusätzlich die Befugnisse, alle Mitglieder des CSM zu bestimmen.

31 Bereits eine sprachliche Besonderheit, da der Begriff „Gewalt“ weder für die Exekutive noch für die Legislative verwendet wird. 
keinerlei personelle Befugnisse, seine Funktionen erschöpfen sich in Gesetzgebung, allgemeiner Rechtspolitik, der Sorge um die gerichtliche Infrastruktur sowie das nichtrichterliche Personal (einschließlich der Staatsanwaltschaft, die in Spanien nicht Teil der rechtsprechenden Gewalt ist). Es besteht daher ein Unterschied zwischen der Justizverwaltung im engeren Sinn, die durch die drei oben genannten Gremien erfolgt, und die „Verwaltung der Gerichtsverwaltung“, die weiterhin der Exekutive anvertraut ist.

\section{Der allgemeine Justizrat}

\section{Zusammensetzung}

Der CGPJ ist Spaniens oberste Justizverwaltungsinstanz. Institutionell verankert ist er seit 1978 in Art. 122 Abs. 2 der spanischen Verfassung. In seine Zuständigkeit fallen alle Laufbahnentscheidungen (z.B. Ernennung und Beförderung) sowie die Disziplinargewalt über die Richterschaft. Er besteht aus einem Vorsitzenden, dem Präsidenten des Obersten Gerichts, und weiteren 19 bis 20 Mitgliedern, ${ }^{32}$ die vom Parlament (je hälftig durch den Senat und das Abgeordnetenhaus) für die Dauer von fünf Jahren gewählt und vom König ernannt werden. 8 davon werden aus den Reihen erfahrener Juristen (z.B. Anwälte oder Hochschullehrer) bestimmt, die übrigen 12 rekrutieren sich aus der Richterschaft selbst. ${ }^{33}$ Hierdurch erhalten die Richter die absolute Mehrheit im Rat. In die Wahl der richterlichen Mitglieder miteinbezogen sind die Richtervereinigungen, die dem Parlament ihre Kandidaten vorschlagen. Die Beteiligung der Richtervereinigungen hat $\mathrm{zu}$ einer hohen Politisierung der Richterschaft und der Wahl der Ratsmitglieder geführt. Bis 2001 traten die Richter, die sich zur Wahl aufstellten, in ihren Wahlunterlagen offen für eine Partei auf. Seit der Änderung des Wahlmodus stellen sowohl die vier Richtervereinigungen als auch die nichtorganisierten Richter je 18 Kandidaten auf, aus denen die beiden Kammern ihre Mandatsträger wählen. Die Wahl muss mit mindestens 60 \% der Stimmen erfolgen. Da hierzu in der Regel die Beteiligung der Opposition erforderlich ist, soll das Wahlsystem eine möglichst pluralistische Zusammensetzung des Rats garantieren und einen einheitlichen Stimmblock der richterlichen Mitglieder verhindern. ${ }^{34}$

32 Der Rat unterbreitet nach der Wahl seiner Mitglieder dem König einen Vorschlag für die Ernennung seines Vorsitzenden, der gleichzeitig Präsident des Obersten Gerichts wird. Es ist nicht notwendig, dass der Vorgeschlagene bereits Mitglied des Rats ist, daher kann die vollständige Besetzung zwischen 20 und 21 Mitgliedern schwanken.

33 Bis 1985 wurden die richterlichen Mitglieder aus der Mitte der Richterschaft gewählt. Die Änderung des Wahlverfahrens war der Machtübernahme der sozialistischen Mehrheit geschuldet, die hiermit die traditionell hochkonservativen und reformunwilligen Strukturen der Richterschaft aufbrechen wollte. Um eine Erneuerung und Demokratisierung der Justiz herbeizuführen, sollte auch die Zusammensetzung der Richter pluralistischer werden. Zudem unterlag das Wahlverfahren der Befürchtung, dass mit ihm eine von den anderen Gewalten nicht mehr kontrollierbare Justiz entstehen würde, die sich selbst korporalistisch regeneriert. Nun bildet der Gerichtsrat zwar die Mehrheitsverhältnisse im Parlament, nicht aber zwingend die Mehrheitsverhältnisse innerhalb der Richterschaft wider, so dass sich viele Richter nicht durch den Rat vertreten sehen.

34 Vgl. Strecker, Selbstverwaltung der Justiz in Spanien, Betrifft Justiz 1998, S. 346 ff. 


\section{Kompetenzen des CGPJ}

Die Befugnisse des Consejo General umfassen alle Fragen des Richterstatus (Ernennung, Zuweisung und Beförderung) sowie die Disziplinargewalt und die Aus- und Fortbildung der Richter. Er organisiert die Auswahlprüfung (sog. Concursos oder Opposiciones) und weist den ernannten Richtern ihre konkreten Stellen bei Gericht zu. ${ }^{35}$

Die spanische Justiz kennt insgesamt drei Beförderungsstufen: ${ }^{36}$ Das Eingangsamt des Juez, die zweite Laufbahnstufe des Magistrado und die höchste Beförderung zum Richter am Obersten Gericht. Die Ernennung zu den jeweiligen Beförderungsstufen erfolgt auf Vorschlag des Rats durch den König mit Gegenzeichnung des Justizministers. Jeder Richter kann sich auf eine frei werdende Beförderungsstelle bewerben. Bei der Auswahl des Kandidaten durch den Consejo General sind dessen berufliche Erfahrung, die Ergebnisse von Prüfungen bei Fortbildungen an der Richterschule des Rats sowie sein Dienstalter die ausschlaggebenden Faktoren. Alle drei Jahre werden daher vom CGPJ Ranglisten nach diesen Kriterien erstellt und veröffentlicht. Nicht relevant sind für die Beförderungen entgegen dem deutschen System die Beurteilungen der Richter, die zwar durch Inspektoren des Rats an den einzelnen Gerichten erstellt, jedoch nur für Disziplinarverfahren herangezogen werden. Kammervorsitzender und Gerichtspräsident sind keine klassischen Beförderungsstellen. Vorsitzender einer Kammer ist automatisch deren ältestes Mitglied. Die Gerichtspräsidenten werden vom Consejo General auf Zeit (5 Jahre) gewählt. Nach seiner Amtszeit kehrt der Gerichtspräsident wieder in sein zuvor ausgeübtes Amt zurück.

Die Disziplinargewalt des Consejo General wird entweder von einer eigenen Disziplinarkommission oder vom Plenum des Rats ausgeübt, eine Kompetenz des Justizministers besteht nicht mehr.

\section{Die Generalversammlung der Richter}

Ergänzt wird das System der Selbstverwaltung durch die Generalversammlung der Richter, die für jeden Gerichtsbezirk (partido judicial) ${ }^{37}$ zusammentritt und für organisatorische Belange zuständig ist. In der Richterversammlung verwirklicht sich die Idee einer „,internen Verwaltung“ der Gerichte. Sie kann von dessen Präsidenten (sog. Juez Decano, der je nach Größe des Gerichtsbezirks entweder gewählt wird oder der Dienstälteste ist) oder von $10 \%$ der Richter einberufen werden, um Fragen von allgemeinem Interesse (z.B. der Gerichtsausstattung oder der Informationstechnologienutzung) zu erörtern. Beschlüsse der Versammlung haben zwar keine Außenwirkung, binden deren Präsidenten jedoch bei der Verhandlung mit anderen amtlichen Stellen bzw. den Regierungsbehörden. Er übernimmt dann die Vermittlerrolle zwischen Judikative und Exekutive und vertritt die Richter des Bezirks nach außen. In der Generalversammlung

35 Vgl. Manzaranes, Der allgemeine Rat der Gerichtsgewalt, DRiZ 1999, S. $317 \mathrm{ff}$.

36 Vgl. Jürgens, Die Besetzung von Funktionsstellen auf Zeit hat sich in Spanien bewährt, Betrifft Justiz 2007, S. $94 \mathrm{ff}$.

37 Die Gerichtsbezirksebene stellt die erste Instanz der Rechtsprechung dar. Hier werden die Richter als Einzelrichter (in den Bereichen der Zivil-, Arbeits-, Strafgerichtsbarkeit etc.) tätig. 
werden jedoch auch Änderungen des Prozess- bzw. materiellen Rechts erörtert, um sich besser auf veränderte Rechtslagen einstellen zu können..$^{38}$

Zusätzlich zur Generalversammlung gibt es in den einzelnen Gerichtsbezirken auch Bereichsspezifische Versammlungen für die einzelnen Rechtsgebiete. Neben der Erörterung bereichsspezifischer Rechtsprechungsfragen (z.B. Auslegungsfragen bei Gesetzesänderungen) sind sie zuständig für die Erstellung eines Geschäftsverteilungsplans und eines Vertretungsplans, die von den Verwaltungskammern der Oberen Gerichtshöfe genehmigt werden müssen.

\section{Die Verwaltungskammern der Oberen Gerichtshöfe}

Als mittlere Instanz der justiziellen Selbstverwaltung fungieren die Verwaltungskammern der Oberen Gerichtshöfe. Sie bestehen aus den Präsidenten der jeweiligen Gerichte, den Vorsitzenden der einzelnen Kammern, im Falle der regionalen Obergerichte den Präsidenten aller Provinzgerichte der Region sowie der gleichen Anzahl an von ihren Kollegen per Mehrheitswahl bestimmten Richtern. Die Kandidatenlisten werden von den Richtervereinigungen gestellt.

In die Zuständigkeit der Verwaltungskammern fallen kleinere Disziplinarverfahren, die Auswahl von Hilfsrichtern, der Austausch mit Regierungsbehörden über die Ausstattung der Gerichte sowie die Genehmigung der Geschäftsverteilungs- und Vertretungspläne. Gegen die Entscheidungen der Verwaltungskammern können vor dem allgemeinen Justizrat Rechtsmittel eingelegt werden.

\section{Zusammenfassung}

Insgesamt werden für das spanische System wenig bis keine Fehlentwicklungen in der Justiz geschildert. Seit der Reform von 1985, durch welche der Wahlmodus des CGPJ geändert wurde und nun alle Mitglieder des Rats durch das Parlament bestimmt werden, ist die vorher als fehlend kritisierte Rückbindung an den Volkswillen gelungen: Alle Mitglieder des Rats sind seither demokratisch legitimiert. Allerdings hat das Erfordernis der Parlamentswahl und die Einbeziehung der Richtervereinigungen, die insgesamt stärker parteiorientiert ausgerichtet sind als im deutschen Rechtsraum, zu einer Politisierung der Dritten Gewalt geführt, die sie dem Verdacht der Parteilichkeit aussetzt. Die Politisierung der gerichtlichen Verwaltung zeigt sich auch darin, dass 2006 trotz Ablauf der Amtszeit kein neuer Justizrat gewählt werden konnte, da sich Abgeordnetenhaus und Senat nicht parteiübergreifend auf neue Kandidaten einigten. ${ }^{39}$ Die unmittelbare Abhängigkeit des höchsten richterlichen Selbstverwaltungsgremiums von der Parlamentsmehrheit trägt zudem insofern den Einwand des fraglichen Mehrwertes mit sich, da es keinen Unterschied zum ebenfalls parlamentarisch verantwortlichen Justizminister gibt.

38 Vgl. Bayo-Delgado, Praktische Erfahrungen aus der Selbstverwaltung: Das Spanische Modell, KritV 2008, S. 398 ff.

39 Vgl. Jürgens (Fn. 36), S. 95. 
Das System der Selbstverwaltung durch verschiedene Organe und unterschiedliche Ebenen versucht, jedem einzelnen Richter Gestaltungsmacht hinsichtlich der eigenen Tätigkeit zu gewähren und die verwaltungstechnische Macht des Consejo General auf den einzelnen Richter und dessen Unabhängigkeit zu minimieren. Diese Reduzierung seiner Macht hat auch ein seit 2006 im Parlament eingebrachter Gesetzesentwurf zum Ziel, der den CGPJ dezentralisieren und in „Autonome Justizräte“ (Consejos Autonomicos de Justicia) umwandeln möchte, da diese der staatlichen Dezentralisierung besser entsprechen würden.

\section{E. Das dänische Modell}

Das dänische System der Gerichtsverwaltung wurde erst in jüngster Vergangenheit reformiert. Die seit dem Inkrafttreten des „Gerichtsverwaltungsaktes“ am 1. Juli 1999 neu geschaffenen Institutionen des Gerichtsverwaltungsrats und des Richterernennungsrats übernehmen von nun an Funktionen, die zuvor beim Justizminister angesiedelt waren. Hierdurch soll die in der dänischen Verfassung in $\S 64$ garantierte richterliche Unabhängigkeit auch strukturell abgesichert werden. $\S 3$ i.V.m. $\S 61$ regelt hierzu nun, dass die Befugnis zur Verwaltung der Justiz bei den Gerichten liege und dass Judikative und Exekutive zu trennen seien. ${ }^{40}$

Grund für die Reform des Gerichtswesens war die Anfang der 90er Jahre immer lauter werdende Kritik an der Macht des Justizministeriums über die Gerichte. Die dänischen Gerichte standen seit 1848 unter der Verwaltung des Justizministeriums und waren finanziell und administrativ von ihm abhängig. Zuständig für die Ernennung und Beförderung der Richter war der Justizminister. Diese personelle Abhängigkeit wurde noch dadurch erhöht, dass Kandidaten für ein Richteramt nach Bestehen der Abschlussprüfung zunächst mindestens 15 Jahre als sogenannte „Bevollmächtigte“ in der Verwaltung, unter anderem im Justizministerium, tätig sein mussten. Nur die Disziplinargewalt oblag und obliegt einem unabhängigen, aus Richtern der verschiedenen Instanzen bestehenden Sondergericht. 1992 führte die umstrittene Handhabung des Ausländergesetzes zum Rücktritt der gesamten dänischen Regierung und eröffnete erneut die Debatte um eine unabhängige Justiz. Auch das Justizministerium geriet unter Verdacht, bezüglich des Gesetzes Einfluss auf die Gerichte ausgeübt zu haben. Von fachlicher und politischer Seite wurde daher empfohlen, die Gerichte aus der Verwaltung auszugliedern. Diese Gerichtsreform fand 1999 durch den Gerichtsverwaltungsakt ihren Abschluss. Durch ihn wurden der Gerichtsverwaltungsrat und der Richterernennungsrat gegründet. Zwar sind die Gerichte nach wie vor ressortmäßig an das Justizministerium angegliedert, es ist jedoch weder im Verhältnis zu den einzelnen Gerichten noch zum Gerichtsverwaltungsrat oder zu seinem Vorstand weisungsbefugt. ${ }^{41}$

40 Vgl. Voermans/Albers, Councils for the Judiciary in EU Countries, 1999, S. 28.

41 Vgl. Feier, Richterliche Selbstverwaltung in Dänemark, DRiZ 2001, S. 436. 


\section{Der Richterernennungsrat (Dommerudncevelserådet)}

Die Befugnis zur Ernennung von Richtern wurde aus dem Justizministerium ausgegliedert und an einen unabhängigen Richterernennungsrat (Dommerudncevelserådet) übertragen. Zwar erfolgt die Einstellung der Richter - auch des übrigen Justizpersonals immer noch formal durch den Justizminister bzw. die Königin. Dem Justizernennungsrat obliegt hierbei jedoch das Initiativrecht. Die einzige Ausnahme hierzu bildet die Ernennung des Präsidenten des Obersten Gerichtshofs, den die Richter aus ihrer Mitte wählen. Der Justizminister folgt den ihm unterbreiteten Ernennungs- und Beförderungsvorschlägen trotz fehlender rechtlicher Bindungswirkung in der Praxis ausnahmslos, eine Abweichung müsste er vor dem Parlament (folketing) rechtfertigen.

Der Rat besteht aus sechs Mitgliedern. Zu ihnen zählen ein Richter des Obersten Gerichtshofs (Oberlandesgericht/hojesteretsdommer), der zugleich als Vorsitzender fungiert, ein Richter am Landgericht (landsdommer), der als Vertreter bestimmt wird, ein Richter der unteren Gerichtsinstanz (Amtsrichter/byrestdommer) sowie ein von der Rechtsanwaltskammer gewählter Anwalt und zwei die Öffentlichkeit repräsentierende nicht-richterliche Mitglieder. Die beiden letzteren werden von der Dänischen Erwachsenen-Bildungsgesellschaft und dem Nationalen Verband der Gemeinden bestimmt. ${ }^{42}$

\section{Der Gerichtsverwaltungsrat (Domstolsstryrelsen)}

Weiter wurde ein Gerichtsverwaltungsrat (Domstolsstryrelsen) eingerichtet, der alle Gerichte in Dänemark zentral verwalten soll; eine Aufgabe, die bisher ebenfalls in den Händen des Justizministers lag und nun eine Loslösung der Gerichtsverwaltung aus dem Einflussbereich der Exekutive erreichen soll. Der Gerichtsverwaltungsrat besteht aus einem Präsidium (bestyrelsen), dem die übergeordnete Leitung obliegt, und einem Direktor mit eigenem Personal, der die Geschäftsführung übernimmt und vom Vorstand eingesetzt (und wieder entlassen) wird. Er besteht aus vier Abteilungen: dem Personalbüro, das für das Personal der Gerichte zuständig ist, einer IT-Abteilung, einer Finanzabteilung und einer Verwaltungsabteilung. Die Verwaltung der einzelnen Gerichte wird durch den zentral agierenden Rat nicht betroffen und bleibt weiterhin in deren Befugnis.

Das Präsidium des Gerichtsverwaltungsrats besteht aus insgesamt elf Mitgliedern, wobei bei der Gründung des Rats darauf Wert gelegt wurde, dass Angehörige der Gerichtsbarkeit mit 8 Vertretern die absolute Mehrheit bilden. Fünf davon stammen aus der Richterschaft selbst (ein Richter des Obersten Gerichtshofes, zwei Appellationsrichter und zwei Amtsrichter als Vertreter der ersten Gerichtsinstanz, so dass alle Ebenen der Gerichtsbarkeit in die Verwaltung mit einbezogen sind). Hinzu kommen ein „Hilfsrichter" als Vertreter des Justizpersonals und zwei Gerichtsbeamte als Repräsentanten des Verwaltungspersonals an den Gerichten. Als weitere Mitglieder sind ein Rechtsanwalt und zwei Personen mit speziellen Verwaltungserfahrungen bzw. Führungs- und Sozialkompetenzen vorgesehen. Die Vorstandsmitglieder werden für einen Zeitraum von vier Jahren benannt und erfüllen diese Aufgabe neben ihrer normalen Tätigkeit.

42 Vgl. Evers-Vosgerau, Die neue Selbstverwaltung der Richter in Dänemark, DRiZ 2000, S. 299. 
Allerdings dürfen die Mitglieder während ihrer Amtszeit nicht gleichzeitig ein Abgeordnetenmandat ausüben oder in der Verwaltung bzw. Regierung tätig sein.

Die Mitglieder des Präsidiums werden formal vom Justizminister ernannt, sie werden jedoch von verschiedenen, von der Exekutive unabhängigen Institutionen bestimmt. So werden die richterlichen Mitglieder aus der Mitte der Richterschaft gewählt und der dem Präsidium angehörige Rechtsanwalt wird von der dänischen Anwaltskammer entsandt. Die zwei Mitglieder, die nicht der Justiz angehören, werden durch öffentliche Institutionen, namentlich die Dänische Universitätsrektoren-Konferenz und die Dänische Nationale Arbeitsmarktrat, ernannt.

Gemäß dem Gerichtsverwaltungsakt besteht die Aufgabe des Gerichtsverwaltungsrats in der Sorge um einen verantwortlichen und zweckmäßigen Betrieb der Gerichte, er hat hierfür die notwendigen Initiativen zu ergreifen. Ihm wird das Budgetantragsrecht für die Gerichtsbarkeit verliehen, d.h. er ist zuständig für die Haushaltsentwürfe des jeweiligen Haushaltsjahrs, für die Verhandlungen mit dem Finanzministerium und die übergeordnete Verteilung der Mittel im Rahmen des erreichten Budgets. In den ersten Jahren seines Bestehens ist es dem Rat entgegen manchen Erwartungen gelungen, ausreichende Mittel für den Betrieb der Gerichte und die Initiierung neuer Projekte (wie eine Änderung des bestehenden EDV-Systems) zu erhalten. ${ }^{43}$ Diese neugewonnene Haushaltsverantwortung bedeutet jedoch auch, dass die Gerichte unter Druck geraten, den Haushaltsantrag untermauernde Statistiken für den Personal- und Sachbedarf zu liefern. Dem Finanzministerium werden auf diese Weise neue Möglichkeiten der Einflussnahme gegeben (so kann es Bedingungen für die gewünschte Mittelzuweisung aufstellen, welche die Art und Weise der Arbeitserledigung betreffen können), so dass auch der neue Gerichtsverwaltungsrat in die Zwickmühle zwischen Effizienz und qualitativer Justizgewährleistung gerät. Der Rat ist weiterhin verantwortlich für die Gerichtsorganisation, z.B. für die Einführung neuer Informationssysteme oder die Übernahme von privatwirtschaftlichen Managementprogrammen (Neue Steuerungsmodelle) für den Justizbereich. ${ }^{44}$

\section{Zusammenfassung}

Wie in anderen nordischen Ländern sind die beiden „Selbstverwaltungsorgane“ des dänischen Justizsystems weder in ihrer Stellung noch in ihren Befugnissen mit denen eines Obersten Richterrats vergleichbar. Ihre Aufgaben sind überwiegend auf finanzielle und administrative Funktionen begrenzt. Von Selbstverwaltungsorganen der Justiz kann daher nur unter Vorbehalt gesprochen werden.

\section{F. Das britische Modell}

Zuletzt soll ein Blick auf das Land geworfen werden, dessen Justizsystem sich vor allem durch die jüngste Reform des Constitutional Reform Acts im Jahre 2005 im Umbruch

43 Es besteht jedoch das Recht des Justizministers, das komplette Präsidium zu entlassen, wenn ihm offensichtliche Fehlplanungen bei der Haushaltsführung zu Last gelegt werden können.

44 Vgl. Oberto (Fn. 1), S. 210. 
befindet und das sich mit der Einrichtung einer unabhängigen Judicial Appointments Commission der europäischen Tendenz hin zu einem eigenständigen Gerichtsverwaltungsgremium anschließt; auch wenn seine Befugnisse - ähnlich dem dänischen Modell - weit hinter denen südeuropäischer Räte zurückbleiben. Zum besseren Verständnis der aktuellen Ausgestaltung der Gerichtsverwaltung soll zunächst die vor 2005 bestehende Rechtslage erläutert werden.

\section{Die englische Gerichtsverwaltung vor dem Constitutional Reform Act}

Bis zu den jüngsten Reformen oblagen alle Befugnisse, welche die Verwaltung der Gerichte betrafen (Ernennung, Beförderung und das Disziplinarrecht), in den Händen des Lord Chancellors (Lordkanzlers), der als Oberster Richter des Landes, als Sprecher des Oberhauses und Mitglied des Kabinetts alle drei Gewalten in einer Person vereint und somit sowohl als Personifizierung als auch als lebende Negierung von Gewaltenteilung gilt. Aus diesem Grund wird die Institution des Lordkanzlers auch mit einer Hummel verglichen, „da jener eine ebensolche verfassungsrechtliche Unmöglichkeit darstellt, wie die Hummel eine aerodynamische Unmöglichkeit ist. “45

Die administrative Verwaltung der Gerichte erfolgte maßgeblich durch die dem Lordkanzler unterstellte Behörde, dem Court Service. Die Ernennung der Richter erfolgte vor 2005 mehrheitlich durch den Lordkanzler. Hierbei wurde nach den verschiedenen Richterämtern unterschieden. Gewohnheitsrechtlich richtete sich die Besetzung von höheren Richterstellen nach dem Vorschlag des Premierministers, der vom Lordkanzler allerdings inhaltlich vorbereitet wurde und so inhaltlich beeinflusst werden konnte; formal erfolgte die Ernennung durch die Krone.

Die ca. 1300 Berufsrichter werden hauptsächlich aus dem Kreis der barristers rekrutiert, dem vor den höheren Gerichten plädierenden, renommierten Teil der britischen Anwaltschaft. Aus den Reihen der solicitors, meist auf Beratung und Prozessvorbereitung beschränkte Anwälte, stammt nur ein kleiner Teil der Ausgewählten. Auch hier traf der Lordkanzler die Auswahl, wobei er sich eines eigenen Amtes, des Lord Chancellor's Department, bediente. Dessen Mitarbeiter übernahmen sowohl die Befragung der Bewerber als auch die Einholung der sog. Soundings, der vertraulichen Einschätzung der Qualität des Bewerbers durch Richter oder andere Anwälte. Kritik an diesem Verfahren wurde weniger an der Möglichkeit der Gefährdung der Unabhängigkeit, als neben der fehlenden demokratischen Legitimation - an der Elitenbildung und dem sozialen Ausschluss durch die Art der Richterrekrutierung geübt. Die Konzentration auf den Kreis der barrister und die vergleichsweise späte Berufung der Anwärter würde zudem zu einer eher konservativ verhafteten Richterschaft führen. Hinzukommen in England noch eine hohe Anzahl an Laienrichtern (ca. 30 000), die an magistrates 'courts als justices of peace weite Teile der niederen Strafgerichtsbarkeit ausüben und die tribunals, die in arbeits-, sozial- oder öffentlich-rechtlichen Angelegenheiten urteilen. Auch hier übernahm der Lordkanzler die Auswahl und Ernennung mit Hilfe sog. local advisory committees, die überwiegend aus Friedensrichter bestanden und die Befähigung der Anwärter prüften.

45 Vgl. hierzu die Darstellung bei Schütz, Der ökonomisierte Richter, 2005, S. 121 f. 
In Abwesenheit eines echten Beförderungssystems wurden zumindest in diesem Bereich keine Einflussmöglichkeiten auf die Rechtsprechung gesehen.

$\mathrm{Zu}$ erwähnen bleibt die Möglichkeit der Entfernung von Richtern aus dem Amt (was in der Funktion der Ausübung von Disziplinargewalt entspricht), die nur bei fehlendem good behaviour durch die beiden Häuser des Parlaments erfolgen kann. Diese in der Praxis faktisch bestehende Unversetzbarkeit gilt jedoch ausschließlich für die obere Richterschaft. Die Richter unterer Gerichtsinstanzen konnten vom Lordkanzler wegen gross misconduct abgesetzt werden, für magistrates galt dies sogar ohne Begründung. Hieraus resultiert eine merkliche Zweiteilung der Richterschaft bezüglich ihrer Unabhängigkeitsgarantie. ${ }^{46}$

\section{II. Änderungen der Gerichtsverwaltung durch den Constitutional Reform Act (2005)}

Durch den Constitutional Reform Act sollte die unter Premierminister Blair angestoßene Modernisierung Englands in Richtung eines modernen Verfassungsstaats vorangetrieben werden. Bereits 2003 wurde, um den Vorgaben des Art. 6 Abs. 1 EMRK nach einer unabhängigen Rechtsprechung gerecht zu werden, ${ }^{47}$ das Lord Chancellor's Department in das Department for Constitutional Affairs umgewandelt und der amtierende Lordkanzler zugleich als Minister for Constitutional Affairs eingesetzt. Zudem wurden court boards eingerichtet, die den Lordkanzler bei der Verwaltung der Gerichte überwachen und kontrollieren sollten.

Das 2005 in Kraft getretene Reformgesetz normiert nun zum ersten Mal explizit das Prinzip der richterlichen Unabhängigkeit. ${ }^{48}$ Es veränderte die Verteilung der Verantwortung für die Gerichtsorganisation grundlegend. Zwar wurde das Amt des Lordkanzlers beibehalten, aber seine gewaltenübergreifenden Kompetenzen beschnitten. Zudem wurde die Judicial Appointments Commission eingerichtet, die bei der Richterernennung mitwirken soll.

\section{Beschneidung der Macht des Lordkanzlers}

Auch wenn in der letzten Hälfte des 20. Jahrhunderts keine Einwirkung des Lordkanzlers auf die Justiz nachweisbar war, konnte und sollte das Grundproblem seiner Zwitterstellung nicht länger geleugnet werden. Es bestand darin, dass die Unabhängigkeit der Justiz von den persönlichen Fähigkeiten des Lordkanzlers abhing, den Balanceakt der Funktionentrennung zu wahren. Durch das Reformgesetz wurden daher seine Befugnisse die Verwaltung und das Personalwesen der Justiz betreffend fast vollständig aufgehoben. Zwar blieb sein Amt bestehen, es ist im Kern aber auf die Befugnisse eines klassischen Justizministers (sog. Secretary of State for Justice) beschränkt worden; das Department

46 Vgl. hierzu Wittreck (Fn. 19), S. 587 ff.

47 Gemäß Art. 6 Abs. 1 EMRK kann nicht mehr von einem unabhängigen und unparteiischen Gericht gesprochen werden, wenn es innerhalb der Gerichtsorganisation zu einer Vermischung von legislativen und judikativen Funktionen kommt.

48 In common law-Ländern übernimmt die konstitutionelle Gesetzgebung oder Rechtsprechung die Funktion einer geschriebenen Verfassung. 
of Constitutional Affairs wurde aufgelöst und in das Ministry of Justice überführt. Es besteht nur noch ein formaler Zusammenhang zwischen dem Justizministerium und der neuen Justizverwaltung, da die Mittel für die Gerichte weiterhin aus dem Budget des Ministeriums stammen.

\section{Der Lord Chief Justice und das Judicial Executive Board}

Statt des Lordkanzlers wurde nun das Amt des Lord Chief Justice (dem hinter dem Lordkanzler zweithöchsten Richter des Landes) zum President of the Courts of England and Wales (Oberhaupt der gesamten Gerichtsbarkeit von England und Wales) aufgewertet, der die Befugnisse zur Vertretung der Richterschaft gegenüber anderen Staatsorganen, zur Ausbildung der Richter und zur Geschäftsverteilung erhält. Hinzu kommen neue Disziplinarbefugnisse, bei denen er jedoch auf die Zustimmung des Lordkanzlers angewiesen ist, sowie das Recht, Budgetverhandlungen mit der Regierung zu führen und die Richtlinien für die Haushaltsplanung der Gerichte aufzustellen. ${ }^{49}$

Da der Lord Chief Justice neben diesen Aufgaben noch den Vorsitz bei ausgewählten Gerichtsverhandlungen führt, wurde ein Judicial Executive Board gebildet, dessen Vorsitzender er ist und das ihn bei seinen Aufgaben unterstützen soll. Es besteht aus sieben vorsitzenden Richtern der höheren Gerichtsbarkeit und deren Stellvertretern und führt die Verwaltungstätigkeiten aus, die vom Lord Chief Justice an sie delegiert wurden.

\section{Judicial Appointments Commission}

Die Judicial Appointments Commission hat die Aufgabe, Kandidaten für ein Richteramt an Hand eines formalisierten Verfahrens auszuwählen. Ausschlaggebend hierbei soll die fachliche Qualifikation der Bewerber sein. Die ausgewählten Kandidaten können jedoch durch den Lordkanzler abgelehnt werden. Er ist allerdings verpflichtet, den dritten Vorschlag, den ihm die Judicial Appointments Commission präsentiert, anzunehmen, so dass bereits rechtlich die Empfehlungen der Kommission vom Kanzler nur begrenzt in Frage gestellt werden können. Erneut kann ein zurückgewiesener Kandidat im selben Wahlgang jedoch nicht von der Kommission durchgesetzt werden. Zudem ist die Judicial Appointments Commission für die Ernennung des Lord Chief Justice verantwortlich. Hierbei ist sie jedoch auf den Vorschlag des Lordkanzlers angewiesen, der auch die Kandidaten für die Besetzung der jeweils höchsten Fachgerichte vorschlägt, die wiederum Mitglieder des Judicial Executive Boards werden.

Die Judicial Appointments Commission, deren Zusammensetzung und Mehrheitsverteilung lange umstritten war, besteht aus 15 Mitgliedern: fünf Berufsrichtern, zwei Mitgliedern, die sich aus der Rechtsanwaltschaft rekrutieren (je ein solicitor und ein barrister), einem Laienrichter sowie einem Mitglied eines tribunal und fünf weiteren nichtrichterlichen Mitgliedern (lay members), so dass die richterlichen Mitglieder nicht mehrheitlich vertreten sind. Der Vorsitz wird von einem Laien ausgeübt. Alle Mitglieder werden auf Vorschlag des Lordkanzlers von der Königin ernannt. Drei der fünf rich-

49 Vgl. Sir Thomas, Einige Perspektiven für Justizverwaltungsräte (Councils fort he Judiciary), KritV 2008, S. 393 f. 
terlichen Mitglieder können vom Lordkanzler nur auf begründeten Vorschlag des Judges ' Council (dem britischen Pendant einer Interessensvertretung der Richterschaft) nominiert werden. Bei der Auswahl der Mitglieder der übrigen Gruppen greift ein komplexes Geflecht von Vorschlags- und Mitwirkungsrechten vierköpfiger panels, die der Lordkanzler zur Benennung von geeigneten Kandidaten bestimmt. Er ist dabei wiederum an Vorschlagsrechte des Lord Chief Justice gebunden. Weitere Befugnisse sind der Kommission nicht verliehen. Kontrolliert wird die Judicial Appointments Commission durch die Judicial Appointments Conduct and Ombudsmen, die ebenfalls vom Lordkanzler eingesetzt werden. ${ }^{50}$

\section{Zusammenfassung}

Es ist festzustellen, dass durch die Beschneidung der Kompetenzen des Lordkanzlers und der Einrichtung der Judicial Appointments Commission zumindest formal die strukturellen Voraussetzungen für eine unabhängige Rechtsprechung und die Diversität der Zusammenstellung der Richterschaft verbessert wurden. De facto bestehen jedoch durch das Amt des Lordkanzlers immer noch weitreichende Einflussmöglichkeiten (z.B. bei der Zusammensetzung der Judicial Appointments Commission und damit indirekt auch bei der Bestimmung des Lord Chief Justices und des Judicial Executive Boards). Allerdings übt der Lordkanzler weiterhin sein Amt mit höchster Zurückhaltung und traditionell gewachsener Achtung vor der richterlichen Unabhängigkeit aus: Von den ca. 450 Richtern, die die Judicial Appointments Commission 2007/2008 ausgewählt haben, wurden alle Ernennungsempfehlung vom Lordkanzler angenommen. Die Judicial Appointments Commission gleicht zwar in ihren Befugnissen eher den deutschen Richterwahlausschüssen, wodurch sie - auch im Hinblick auf die Mehrheitsverhältnisse bei der Zusammensetzung - nur eingeschränkt als richterliches Selbstverwaltungsgremium bezeichnet werden kann. Durch sie wurden jedoch die Transparenz und das öffentliche Vertrauen in den Richterauswahlprozess weiter gestärkt - obwohl er bereits zuvor aufgrund der gewachsenen Rechtstradition stark vom Geist der Unabhängigkeit geprägt war.

50 Vgl. Wittreck (Fn. 19), S. 599 ff. 